\title{
MISCELLANEOUS DYNAMIC EQUATIONS *
}

\author{
ELVAN AKIN-BOHNER ${ }^{\dagger}$ AND MARTIN BOHNER ${ }^{\dagger}$
}

\begin{abstract}
We consider several dynamic equations and present methods on how to solve these equations. Among them are linear equations of higher order, Euler-Cauchy equations of higher order, logistic equations (or Verhulst equations), Bernoulli equations, Riccati equations, and Clairaut equations. In order to solve Bernoulli dynamic equations, we define an important product on the set of positively regressive functions and give a power rule in terms of this product.
\end{abstract}

1. Introduction. The calculus on time scales has been introduced in order to unify the theories of differential equations and of difference equations and in order to extend those theories to a more general class of so-called dynamic equations. For an introduction into the calculus of time scales we refer to $[3,4,5]$. Here we just would like to mention that any nonempty, closed subset $\mathbb{T}$ of $\mathbb{R}$ is called a time scale, and that the forward jump operator $\sigma: \mathbb{T} \rightarrow \mathbb{T}$ is defined by $\sigma(t)=\inf \{s \in \mathbb{T}: s \geq t\}$. Similarly, a backward jump operator $\rho$ is defined, and the graininess $\mu$ of $\mathbb{T}$ is given by $\mu(t)=\sigma(t)-t$. In this paper we consider certain classes of simple dynamic equations and present methods on how to solve them. Discrete versions of some of those equations are studied in [1, Chapter 3].

First, in Section 2, we consider higher order linear dynamic equations with constant coefficients, i.e., equations of the form

$$
\sum_{k=0}^{n} \alpha_{k} y^{\Delta^{k}}=0
$$

For a reader not familiar with the time scales calculus, it is helpful (before studying [3]) to think of the operator ${ }^{\Delta}$ as the usual derivative if $\mathbb{T}=\mathbb{R}$ and the usual forward difference operator if $\mathbb{T}=\mathbb{Z}$. Of course, as in the theory of ordinary differential equations, the so-called characteristic polynomial plays a central rôle, and in particular the case of multiple roots of the characteristic polynomial will yield some interesting results. Using a similar method, in Section 3 we carry the study of Euler-Cauchy equations from the second order case as presented in [3, Section 3.7] to the higher order case. However, in the higher order case we are forced - due to the fact that forward jump operators are not necessarily differentiable - to factor those EulerCauchy equations appropriately, namely as

$$
\sum_{k=0}^{n} \alpha_{k} M_{k} y=0
$$

where the operators $M_{k}$ are defined recursively by

$$
M_{0} y=y \quad \text { and } \quad M_{k+1} y=t\left(M_{k} y\right)^{\Delta} \text { for } k \in \mathbb{N}_{0} .
$$

As in the higher order linear case, an associated characteristic polynomial is important, and as for linear equations, the case of multiple roots of this characteristic polynomial turns out to be of particular interest.

\footnotetext{
*Received November 16, 2002; accepted for publication May 19, 2003.

${ }^{\dagger}$ University of Missouri-Rolla, Department of Mathematics and Statistics, Rolla, MO 65401, USA (akine@umr.edu,bohner@umr.edu).
} 
Motivated by the fact that (for ordinary differential equations) the reciprocal of a solution of a linear equation is a solution of a logistic equation, we introduce our two versions - two, as there are two versions of linear equations (denote $v^{\sigma}=v \circ \sigma$ )

$$
u^{\Delta}=p(t) u+f(t) \quad \text { and } \quad v^{\Delta}=-p(t) v^{\sigma}+f(t)
$$

- of logistic equations. These logistic equations will appear in the forms

$$
y^{\Delta}=[\ominus(p(t)+f(t) y)] y \quad \text { and } \quad x^{\Delta}=[p(t) \ominus(f(t) x)] x,
$$

where $\ominus$ is defined as follows: The set $\mathcal{R}$ of all complex-valued, regressive (i.e., $1+$ $\mu(t) p(t) \neq 0$ for all $t \in \mathbb{T}$ ), and rd-continuous (i.e., functions in $\mathrm{C}_{\mathrm{rd}}$, i.e., continuous at points $t$ with $\sigma(t)=t$ and left-sided limits exist and are finite at points $t$ with $\rho(t)=t$ ) functions is an Abelian group (the so-called regressive group) under the addition $\oplus$ defined by $p \oplus q=p+q+\mu p q$, and $\ominus p$ is the additive inverse of $p \in \mathcal{R}$, i.e., $\ominus p=-p /(1+\mu p)$ (see [3, Exercise 2.26]). Now, knowledge of solutions of the linear equations (1.4) then enables us to exhibit solutions of the logistic equations (1.5). Next, we are aiming to introduce Bernoulli equations in such a way that their solutions raised to the $\alpha$ th power are solutions of logistic equations (1.5). However, in order to do so, we first need to use the chain rule [3, Theorem 1.90] for finding a simple form of the derivative of $y^{\alpha}$. This leads to the introduction of a circle dot multiplication $\odot$ defined by

$$
(\alpha \odot p)(t):=\alpha p(t) \int_{0}^{1}(1+\mu(t) p(t) h)^{\alpha-1} d h
$$

which then may be used to study Bernoulli equations of the form

$$
x^{\Delta}=\left[p(t) \ominus\left(\frac{1}{\alpha} \odot\left(f(t) x^{\alpha}\right)\right)\right] x,
$$

and solutions of Bernoulli equations (see Section 6) then have our above described desired property, so that they can readily be found as we know solutions of the logistic equations (1.5). Furthermore, the circle dot product $\odot$ turns out to be interesting in itself, and we devote Section 5 of this paper to the study of this product. Interesting properties of this product are the formulas

$$
\frac{\left(x^{\alpha}\right)^{\Delta}}{x^{\alpha}}=\alpha \odot \frac{x^{\Delta}}{x}, \quad 1+\mu(\alpha \odot p)=(1+\mu p)^{\alpha}, \quad \text { and } \quad e_{\alpha \odot p}=e_{p}^{\alpha} .
$$

Note that for $p \in \mathcal{R}$, the unique solution of $y^{\Delta}=p(t) y$ satisfying $y\left(t_{0}\right)=1$ (see [3, Theorem 2.33]) is denoted as $e_{p}\left(\cdot, t_{0}\right)$, and the third formula in (1.8) nicely supplements the known relations (see [3, Theorem 2.36], and this is essentially a consequence of the product rule $(p q)^{\Delta}=p^{\Delta} q+p^{\sigma} q^{\Delta}$ and the quotient rule $\left.(p / q)^{\Delta}=\left(p^{\Delta} q-p q^{\Delta}\right) /\left(q q^{\sigma}\right)\right)$

$$
e_{p} e_{q}=e_{p \oplus q} \quad \text { and } \quad \frac{e_{p}}{e_{q}}=e_{p \ominus q} .
$$

Moreover, the set of real-valued, positively regressive functions $\mathcal{R}^{+}$, i.e., the set of all rd-continuous functions $p$ that satisfy $1+\mu(t) p(t)>0$ for all $t \in \mathbb{T}$ (see $[3$, Definition $2.45]$ ), together with addition $\oplus$ and (real) scalar multiplication $\odot$, turns out to be a vector space, which we call the regressive vector space. 
In Section 7 we also consider Riccati equations of the form

$$
z^{\Delta}+q(t)+r(t) z^{\sigma}+p(t)\left(\frac{z}{p(t)}\right)^{(2)}=0
$$

where the circle square of $x \in \mathcal{R}$ is defined to be $x^{(2)}=(-x)(\ominus x)$. Properties of the circle square are given in [3, Theorem 2.19], and we use them to relate solutions of the Riccati equation to solutions of the logistic equations (1.5), provided one particular solution of the Riccati equation is known. Finally, in Section 8 we study Clairaut equations appearing as

$$
y=t y^{\Delta}+f\left(y^{\Delta}\right)
$$

where $f: \mathbb{R} \rightarrow \mathbb{R}$ may be any continuously differentiable function.

Note that dynamic equations on time scales (also on time scales different from $\mathbb{R}$ or $\mathbb{Z}$ ) have many applications (see $[3$, Section 1.3$]$ ). A recent paper of F. M. Atıc1 and M. Atıc1 [2] discusses some applications to complexity of algorithms, where many equations like $T(n)=3 T(n / 2)+n \log n, T(n)=5 T(n / 5)=n / \log n, T(n)=3 T(n / 3+$ $5)+n / 2, T(n)=T(n-1)+1 / n$, and $T(n)=\sqrt{n} T(\sqrt{n})+n$ arise. All these equations can be rewritten as dynamic equations on particular time scales and can be treated with the methods presented in this paper.

2. Linear Equations. We consider homogeneous linear dynamic equations of higher order with constant coefficients (1.1), where $y^{\Delta^{k}}$ are defined recursively by

$$
y^{\Delta^{0}}=y \quad \text { and } \quad y^{\Delta^{k+1}}=\left(y^{\Delta^{k}}\right)^{\Delta} \text { for } k \in \mathbb{N}_{0}
$$

and $\alpha_{k} \in \mathbb{C}$ for all $0 \leq k \leq n$ with $\alpha_{n} \neq 0$. We call

$$
\varphi(\lambda)=\sum_{k=0}^{n} \alpha_{k} \lambda^{k}
$$

the associated characteristic polynomial.

THEOREM 2.1. If $\lambda \in \mathcal{R}$ is a zero of $(2.1)$, then $e_{\lambda}\left(\cdot, t_{0}\right)$ solves (1.1).

Proof. According to our assumptions, $\lambda \in \mathcal{R}$, and hence $y(t)=e_{\lambda}\left(t, t_{0}\right)$ is well defined. We find $y^{\Delta^{k}}=\lambda^{k} y$ for all $k \in \mathbb{N}_{0}$, and therefore

$$
\sum_{k=0}^{n} \alpha_{k} y^{\Delta^{k}}=\sum_{k=0}^{n} \alpha_{k} \lambda^{k} y=\varphi(\lambda) y .
$$

Hence, if $\varphi(\lambda)=0$, then $y$ solves (1.1).

Definition 2.2. Equation (1.1) is called regressive if $\lambda \in \mathcal{R}$ for all zeros $\lambda$ of $\varphi$.

THEOREM 2.3. Equation (1.1) is regressive if and only if

$$
\sum_{k=0}^{n} \alpha_{k}(-\mu(t))^{n-k} \neq 0 \quad \text { for all } \quad t \in \mathbb{T} .
$$



Then

Proof. To show this, let $\lambda_{1}, \lambda_{2}, \ldots, \lambda_{n}$ be the zeros of $\varphi$ counting multiplicities.

$$
\varphi(\lambda)=\sum_{k=0}^{n} \alpha_{k} \lambda^{k}=\alpha_{n} \prod_{\nu=1}^{n}\left(\lambda-\lambda_{\nu}\right) .
$$

The condition $\lambda_{\nu} \in \mathcal{R}$ for all $1 \leq \nu \leq n$ is equivalent to $\prod_{\nu=1}^{n}\left(1+\mu(t) \lambda_{\nu}\right) \neq 0$. If $\mu(t)=0$, then $\alpha_{n} \prod_{\nu=1}^{n}\left(1+\mu(t) \bar{\lambda}_{\nu}\right)=\alpha_{n}=\sum_{k=0}^{n} \alpha_{k}(-\mu(t))^{n-k}$. If $\mu(t) \neq 0$, then

$$
\begin{aligned}
\alpha_{n} \prod_{\nu=1}^{n}\left(1+\mu(t) \lambda_{\nu}\right) & =\alpha_{n}(-\mu(t))^{n} \prod_{\nu=1}^{n}\left[\left(-\frac{1}{\mu(t)}\right)-\lambda_{\nu}\right] \\
& =(-\mu(t))^{n} \varphi\left(-\frac{1}{\mu(t)}\right) \\
& =(-\mu(t))^{n} \sum_{k=0}^{n} \alpha_{k}\left(-\frac{1}{\mu(t)}\right)^{k} \\
& =\sum_{k=0}^{n} \alpha_{k}(-\mu(t))^{n-k} .
\end{aligned}
$$

This proves our result.

REMARK 2.4. Let $\lambda_{1}, \lambda_{2}, \ldots, \lambda_{n}$ be the zeros of the characteristic polynomial (2.1). Then it is easy to see that (1.1) is regressive iff

$$
\bigoplus_{i=1}^{n} \lambda_{i} \in \mathcal{R}, \quad \text { where } \quad \bigoplus_{i=1}^{n} \lambda_{i}:=\lambda_{1} \oplus \lambda_{2} \oplus \ldots \oplus \lambda_{n}
$$

ExAmple 2.5. Suppose $\mathbb{T}=2^{\mathbb{N}_{0}}$ and consider the equation

$$
y^{\Delta \Delta}-3 y^{\Delta}+2 y=0 .
$$

The characteristic polynomial for $(2.3)$ is $\lambda^{2}-3 \lambda+2$ and hence has zeros 1 and 2 . The regressivity condition $(2.2)$ for $(2.3)$ reads $2 \mu^{2}(t)+3 \mu(t)+1 \neq 0$ for all $t \in \mathbb{T}$, and it is clearly satisfied. Hence two solutions (compare [3, Example 2.55]) of (2.3) are

$$
y_{1}(t)=e_{1}(t, 1)=\prod_{k=0}^{\log _{2} t-1}\left(1+2^{k}\right) \quad \text { and } \quad y_{2}(t)=e_{2}(t, 1)=\prod_{k=1}^{\log _{2} t}\left(1+2^{k}\right),
$$

where the empty product is understood to be 1.

Now we consider linear equations in the multiple root case. The following lemma collects some identities, that are useful when trying to solve this problem by the variation of parameters method.

Lemma 2.6. Suppose $\lambda \in \mathcal{R}$, let $p$ be differentiable, and assume that there exists a sequence $\left\{p_{k}\right\}_{k \in \mathbb{N}_{0}}$ satisfying

$$
p_{0}=p \quad \text { and } \quad p_{k}^{\Delta}=\frac{p_{k+1}}{1+\lambda \mu(t)} \text { for } k \in \mathbb{N}_{0} .
$$


Let $y(t)=p(t) e_{\lambda}\left(t, t_{0}\right)$. Then we have

$$
y^{\Delta^{k}}=\left\{\sum_{\nu=0}^{k}\left(\begin{array}{l}
k \\
\nu
\end{array}\right) p_{\nu} \lambda^{k-\nu}\right\} e_{\lambda}
$$

for all $k \in \mathbb{N}_{0}$ and

$$
\sum_{k=0}^{n} \alpha_{k} y^{\Delta^{k}}=\left\{\sum_{\nu=0}^{n} p_{\nu} \frac{\varphi^{(\nu)}(\lambda)}{\nu !}\right\} e_{\lambda}
$$

Proof. We show (2.5) by induction. First $y^{\Delta^{0}}=y=p e_{\lambda}=p_{0} e_{\lambda}$, and so (2.5) holds for $k=0$. If $(2.5)$ holds for some $k \in \mathbb{N}_{0}$, then (use the product rule)

$$
\begin{aligned}
& y^{\Delta^{k+1}}=\left(\left\{\sum_{\nu=0}^{k}\left(\begin{array}{l}
k \\
\nu
\end{array}\right) p_{\nu} \lambda^{k-\nu}\right\} e_{\lambda}\right)^{\Delta} \\
& =\sum_{\nu=0}^{k}\left(\begin{array}{l}
k \\
\nu
\end{array}\right) \lambda^{k-\nu}\left\{p_{\nu} e_{\lambda}^{\Delta}+p_{\nu}^{\Delta} e_{\lambda}^{\sigma}\right\} \\
& =\sum_{\nu=0}^{k}\left(\begin{array}{l}
k \\
\nu
\end{array}\right) \lambda^{k-\nu}\left\{p_{\nu} \lambda e_{\lambda}+p_{\nu}^{\Delta}(1+\mu \lambda) e_{\lambda}\right\} \\
& =\left\{\sum_{\nu=0}^{k}\left(\begin{array}{l}
k \\
\nu
\end{array}\right) p_{\nu} \lambda^{k+1-\nu}+\sum_{\nu=0}^{k}\left(\begin{array}{l}
k \\
\nu
\end{array}\right) p_{\nu+1} \lambda^{k-\nu}\right\} e_{\lambda} \\
& =\left\{p_{0} \lambda^{k+1}+\sum_{\nu=1}^{k}\left[\left(\begin{array}{l}
k \\
\nu
\end{array}\right)+\left(\begin{array}{c}
k \\
\nu-1
\end{array}\right)\right] p_{\nu} \lambda^{k+1-\nu}+p_{k+1}\right\} e_{\lambda} \\
& =\left\{\sum_{\nu=0}^{k+1}\left(\begin{array}{c}
k+1 \\
\nu
\end{array}\right) p_{\nu} \lambda^{k+1-\nu}\right\} e_{\lambda}
\end{aligned}
$$

so that (2.5) holds for $k+1$. This proves that (2.5) holds for all $k \in \mathbb{N}_{0}$. Now

$$
\begin{aligned}
\sum_{k=0}^{n} \alpha_{k} y^{\Delta^{k}} & =\sum_{k=0}^{n} \alpha_{k}\left\{\sum_{\nu=0}^{k}\left(\begin{array}{l}
k \\
\nu
\end{array}\right) p_{\nu} \lambda^{k-\nu}\right\} e_{\lambda} \\
& =\left\{\sum_{\nu=0}^{n} \sum_{k=\nu}^{n} \alpha_{k}\left(\begin{array}{l}
k \\
\nu
\end{array}\right) p_{\nu} \lambda^{k-\nu}\right\} e_{\lambda} \\
& =\left\{\sum_{\nu=0}^{n} \frac{p_{\nu}}{\nu !} \sum_{k=\nu}^{n} \alpha_{k} \frac{k !}{(k-\nu) !} \lambda^{k-\nu}\right\} e_{\lambda} \\
& =\left\{\sum_{\nu=0}^{n} \frac{p_{\nu}}{\nu !} \varphi^{(\nu)}(\lambda)\right\} e_{\lambda},
\end{aligned}
$$

and this proves $(2.6)$.

THEOREM 2.7. Suppose $\lambda \in \mathcal{R}$, and assume that $\lambda$ is a zero of (2.1) with multiplicity at least $m \in \mathbb{N}$. Put $p_{m-1}=1, p_{k}=0$ for all $k \geq m$, and recursively

$$
p_{k}(t)=\int_{t_{0}}^{t} \frac{p_{k+1}(\tau)}{1+\lambda \mu(\tau)} \Delta t \quad \text { for } \quad m-2 \geq k \geq 0 .
$$


Then $y(t)=p(t) e_{\lambda}\left(t, t_{0}\right)$ is a solution of (1.1), where $p=p_{0}$.

Proof. Note that the sequence $\left\{p_{k}\right\}_{k \in \mathbb{N}_{0}}$ defined as above is well defined (only rd-continuous functions are integrated) and clearly satisfies (2.4). Since $\varphi^{(\nu)}(\lambda)=0$ for all $0 \leq \nu<m,(2.6)$ implies that $y(t)=p(t) e_{\lambda}\left(t, t_{0}\right)$ solves $(1.1)$.

EXAmple 2.8. If $\lambda \in \mathcal{R}$ is a triple zero of $\varphi$, then

$$
e_{\lambda}\left(t, t_{0}\right), \quad e_{\lambda}\left(t, t_{0}\right) \int_{t_{0}}^{t} \frac{\Delta \tau}{1+\lambda \mu(\tau)}, \quad \text { and } \quad e_{\lambda}\left(t, t_{0}\right) \int_{t_{0}}^{t} \frac{\int_{t_{0}}^{\tau} \frac{\Delta s}{1+\lambda \mu(s)}}{1+\lambda \mu(\tau)} \Delta \tau .
$$

are three solutions of (1.1). Note that for $\mathbb{T}=\mathbb{R}$ we have

$$
\int_{t_{0}}^{t} \frac{\Delta \tau}{1+\lambda \mu(\tau)}=\int_{t_{0}}^{t} d \tau=t-t_{0}
$$

REMARK 2.9. The assumption $\lambda \in \mathcal{R}$ in this section and throughout is mainly a matter of convenience. It is possible to extend the presented results to the nonregressive case in the following way: If $\lambda \in \mathbb{C}$ is a zero of (2.1) and if we put $t^{*}:=\inf \{t \in \mathbb{T}: 1+\mu(t) \lambda=0\}$ (where $\inf \emptyset=\infty$ ), then $y$ defined by $y(t)=e_{\lambda}\left(t, t_{0}\right)$ for $t \leq t^{*}$ and $y(t)=0$ for $t>t^{*}$ is a solution of (1.1).

3. Euler Equations. Throughout this section we assume that $\mathbb{T}$ is a time scale with $\mathbb{T} \subset(0, \infty)$. Motivated by the fact that solutions of Euler differential equations (i.e., for $\mathbb{T}=\mathbb{R}$ ) are of the form $y(t)=t^{\lambda}$ with $y^{\prime}(t)=\frac{\lambda}{t} y(t)$, we want to look for solutions of the form

$$
y(t)=e_{\frac{\lambda}{t}}\left(t, t_{0}\right)
$$

also in the case of an Euler dynamic equation on an arbitrary time scale. Note that for such $y$ we have

$$
y^{\Delta}(t)=\frac{\lambda}{t} y(t) \quad \text { and } \quad y^{\Delta \Delta}(t)=\frac{\lambda(\lambda-1)}{t \sigma(t)} y(t),
$$

and unfortunately $y^{\Delta \Delta}$ may not be differentiable (see [3, Example 1.56]) again for a general time scale. But note that

$$
\begin{gathered}
t y^{\Delta}(t)=\lambda y(t), \quad\left(t y^{\Delta}(t)\right)^{\Delta}=\frac{\lambda^{2}}{t} y(t), \\
t\left(t y^{\Delta}(t)\right)^{\Delta}=\lambda^{2} y(t), \quad\left(t\left(t y^{\Delta}(t)\right)^{\Delta}\right)^{\Delta}=\frac{\lambda^{3}}{t} y(t), \cdots,
\end{gathered}
$$

and hence we wish to consider equations of the form (1.2), where the operators $M_{k}$ are defined recursively by (1.3). We call (1.2) an Euler equation of $n$th order, and as before (2.1) is called the associated characteristic polynomial. Theorems 3.1 and 3.3 below may be shown in a way completely analogous to the proofs of Theorems 2.1 and 2.3 , and hence we omit their proofs.

THEOREM 3.1. If $\lambda / t \in \mathcal{R}$ and $\lambda$ is a zero of $(2.1)$, then $e_{\frac{\lambda}{t}}\left(\cdot, t_{0}\right)$ solves (1.2). 
Definition 3.2. Equation (1.2) is called regressive if $\frac{\lambda}{t} \in \mathcal{R}$ for all zeros $\lambda$ of $\varphi$.

THEOREM 3.3. Equation (1.2) is regressive if and only if

$$
\sum_{k=0}^{n} \alpha_{k} t^{k}(-\mu(t))^{n-k} \neq 0 \quad \text { for all } \quad t \in \mathbb{T} .
$$

ExAmple 3.4. If $n=2$, then (1.2) can be rewritten as

$$
\alpha_{2} t\left(t y^{\Delta}\right)^{\Delta}+\alpha_{1} t y^{\Delta}+\alpha_{0} y=\alpha_{2}\left[t \sigma(t) y^{\Delta \Delta}+\left(\frac{\alpha_{1}}{\alpha_{2}}+1\right) t y^{\Delta}+\frac{\alpha_{0}}{\alpha_{2}} y\right] .
$$

Hence (1.2) is equivalent to

$$
t \sigma(t) y^{\Delta \Delta}+a t y^{\Delta}+b y=0 \quad \text { with } \quad a=\frac{\alpha_{1}}{\alpha_{2}}+1, b=\frac{\alpha_{0}}{\alpha_{2}} .
$$

The characteristic polynomial of (3.2) has the same zeros as $\lambda^{2}+(a-1) \lambda+b$. The regressivity condition (3.1) for (3.2) is equivalent to

$$
t \sigma(t)-a t \mu(t)+b(\mu(t))^{2} \neq 0 \quad \text { for all } \quad t \in \mathbb{T} .
$$

EXAmple 3.5. If $n \geq 3$, then (1.2) can be "expanded" as in Example 3.4 if $\mathbb{T}$ is a time scale with differentiable forward jump $\sigma$. E.g., if $\mathbb{T}=\mathbb{R}$, then

$$
\alpha_{3} t\left(t\left(t y^{\Delta}\right)^{\Delta}\right)^{\Delta}+\alpha_{2} t\left(t y^{\Delta}\right)^{\Delta}+\alpha_{1} t y^{\Delta}+\alpha_{0} y=0,
$$

which is equivalent to

(3.4) $t^{3} y^{\prime \prime \prime}+a t^{2} y^{\prime \prime}+b t y^{\prime}+c y=0 \quad$ with $\quad a=3+\frac{\alpha_{2}}{\alpha_{3}}, b=1+\frac{\alpha_{1}}{\alpha_{3}}+\frac{\alpha_{2}}{\alpha_{3}}, c=\frac{\alpha_{0}}{\alpha_{3}}$.

Note that the characteristic equation for (3.4) reads

$$
\lambda^{3}+(a-3) \lambda^{2}+(b-a+2) \lambda+c=0
$$

while the characteristic equation for (3.3) reads - in a sense "more natural" and "suggestive" - simply

$$
\alpha_{3} \lambda^{3}+\alpha_{2} \lambda^{2}+\alpha_{1} \lambda+\alpha_{0}=0 .
$$

This remark applies to Euler equations of any order and on any time scale: Characteristic equations for Euler equations in factored form are "easier to remember" than those for the equations in expanded form.

EXAMPLE 3.6. If $\varphi(0)=0$ or $\varphi(1)=0$, then we have (on any time scale)

$$
e_{0}\left(t, t_{0}\right)=1 \quad \text { and } \quad e_{\frac{1}{t}}\left(t, t_{0}\right)=\frac{t}{t_{0}} .
$$


EXAMPLE 3.7. It is easy to verify that

$$
\begin{gathered}
e_{\frac{\lambda}{t}}\left(t, t_{0}\right)=\left(\frac{t}{t_{0}}\right)^{\lambda} \quad \text { if } \quad \mathbb{T}=\mathbb{R}, \\
e_{\frac{\lambda}{t}}\left(t, t_{0}\right)=\frac{\Gamma(t+\lambda)}{\Gamma(t)} \frac{\Gamma\left(t_{0}\right)}{\Gamma\left(t_{0}+\lambda\right)} \quad \text { if } \quad \mathbb{T}=\mathbb{Z},
\end{gathered}
$$

where $\Gamma$ is the gamma function, and

$$
e_{\frac{\lambda}{t}}(t, 1)=t^{\log _{q}[1+(q-1) \lambda]} \quad \text { if } \quad \mathbb{T}=q^{\mathbb{N}_{0}} \text { with } q>1
$$

Now we consider Euler equations in the multiple root case. The following lemma collects some identities that are useful when trying to solve this problem by the variation of parameters method. Its proof is completely analogous to the proof of Lemma 2.6, and therefore we omit it.

Lemma 3.8. Suppose $\frac{\lambda}{t} \in \mathcal{R}$, let $p$ be differentiable, and assume that there exists a sequence $\left\{p_{k}\right\}_{k \in \mathbb{N}_{0}}$ satisfying

$$
p_{0}=p \quad \text { and } \quad p_{k}^{\Delta}=\frac{p_{k+1}}{t+\lambda \mu(t)} \text { for } k \in \mathbb{N}_{0} .
$$

Let $y(t)=p(t) e_{\frac{\lambda}{t}}\left(t, t_{0}\right)$. Then we have

$$
M_{k} y=\left\{\sum_{\nu=0}^{k}\left(\begin{array}{l}
k \\
\nu
\end{array}\right) p_{\nu} \lambda^{k-\nu}\right\} e_{\frac{\lambda}{t}}
$$

for all $k \in \mathbb{N}_{0}$ and

$$
\sum_{k=0}^{n} \alpha_{k} M_{k} y=\left\{\sum_{\nu=0}^{n} p_{\nu} \frac{\varphi^{(\nu)}(\lambda)}{\nu !}\right\} e_{\frac{\lambda}{t}}
$$

As in Theorem 2.7, we now may use Lemma 3.8 to obtain the following result.

TheOrem 3.9. Suppose $\frac{\lambda}{t} \in \mathcal{R}$, and assume that $\lambda$ is a zero of (2.1) with multiplicity at least $m \in \mathbb{N}$. Put $p_{m-1}=1, p_{k}=0$ for all $k \geq m$, and recursively

$$
p_{k}(t)=\int_{t_{0}}^{t} \frac{p_{k+1}(\tau)}{\tau+\lambda \mu(\tau)} \Delta t \quad \text { for } \quad m-2 \geq k \geq 0 .
$$

Then $y(t)=p(t) e_{\frac{\lambda}{t}}\left(t, t_{0}\right)$ is a solution of $(1.2)$, where $p=p_{0}$.

EXAMPLE 3.10. If $\lambda$ is a triple zero of $\varphi$ with $\lambda / t \in \mathcal{R}$, then

$$
e_{\frac{\lambda}{t}}\left(t, t_{0}\right), \quad e_{\frac{\lambda}{t}}\left(t, t_{0}\right) \int_{t_{0}}^{t} \frac{\Delta \tau}{\tau+\lambda \mu(\tau)}, \quad \text { and } \quad e_{\frac{\lambda}{t}}\left(t, t_{0}\right) \int_{t_{0}}^{t} \frac{\int_{t_{0}}^{\tau} \frac{\Delta s}{s+\lambda \mu(s)}}{\tau+\lambda \mu(\tau)} \Delta \tau
$$

are three solutions of (1.2). Note that for $\mathbb{T}=\mathbb{R}$ we have

$$
\int_{t_{0}}^{t} \frac{\Delta \tau}{\tau+\lambda \mu(\tau)}=\int_{t_{0}}^{t} \frac{d \tau}{\tau}=\log \frac{t}{t_{0}} .
$$


EXAMPLE 3.11. Suppose $\lambda$ is a double zero of a characteristic polynomial (2.1) for $\mathbb{T}=q^{\mathbb{N}_{0}}$ (where $q>1$ ) with $\lambda / t \in \mathcal{R}$, and let $t_{0}=1$. Then two solutions of the corresponding Euler $q$-difference equation $(1.2)$ are given by $y_{1}(t)=t^{\log _{q}[1+(q-1) \lambda]}$ (see Example 3.7) and

$$
y_{2}(t)=[1+(q-1) \lambda] y_{1}(t) \int_{1}^{t} \frac{\Delta \tau}{\tau+\lambda \mu(\tau)}=y_{1}(t) \int_{1}^{t} \frac{\Delta \tau}{\tau} .
$$

Note that

$$
\int_{1}^{q^{m}} \frac{\Delta \tau}{\tau}=\sum_{k=0}^{m-1} \int_{q^{k}}^{\sigma\left(q^{k}\right)} \frac{\Delta \tau}{\tau}=\sum_{k=0}^{m-1} \frac{\mu\left(q^{k}\right)}{q^{k}}=\sum_{k=0}^{m-1} \frac{(q-1) q^{k}}{q^{k}}=m(q-1),
$$

and so $y_{2}(t)=(q-1) t^{\log _{q}[1+(q-1) \lambda]} \log _{q} t$. In particular, if $q=2$, then

$$
y_{1}(t)=t^{\log _{2}(1+\lambda)} \quad \text { and } \quad y_{2}(t)=t^{\log _{2}(1+\lambda)} \log _{2} t
$$

EXAMPLE 3.12. Let $\mathbb{T}=\mathbb{N}^{2}$ and consider the equation

$$
t(1+\sqrt{t})^{2} y^{\Delta \Delta}-t y^{\Delta}+y=0 .
$$

Here, $\sigma(t)=(1+\sqrt{t})^{2}$ and $\mu(t)=1+2 \sqrt{t}$. The characteristic polynomial is $\lambda^{2}-2 \lambda+1$. Hence two solutions are given by (see Example 3.6)

$$
y_{1}(t)=e_{\frac{1}{t}}(t, 1)=t \quad \text { and } \quad y_{2}(t)=y_{1}(t) \int_{1}^{t} \frac{\Delta \tau}{\tau+\mu(\tau)}=t \int_{1}^{t} \frac{\Delta \tau}{\sigma(\tau)}
$$

Note that

$$
\int_{1}^{m^{2}} \frac{\Delta \tau}{\sigma(\tau)}=\sum_{k=1}^{m-1} \int_{k^{2}}^{\sigma\left(k^{2}\right)} \frac{\Delta \tau}{\sigma(\tau)}=\sum_{k=1}^{m-1} \frac{\mu\left(k^{2}\right)}{\sigma\left(k^{2}\right)}=m-1-\sum_{k=1}^{m-1}\left(\frac{k}{k+1}\right)^{2}
$$

and hence

$$
y_{2}(t)=t\left[\sqrt{t}-1-\sum_{k=1}^{\sqrt{t}-1}\left(\frac{k}{k+1}\right)^{2}\right]
$$

4. Logistic Equations. Let us first recall the following results from [3, Theorems 2.77 and 2.74].

Theorem 4.1 (Variation of Constants). Suppose $p \in \mathcal{R}$ and $f \in \mathrm{C}_{\mathrm{rd}}$. Let $t_{0} \in \mathbb{T}$.

(i) Let $u_{0} \in \mathbb{R}$. The unique solution $u$ satisfying the left equation in (1.4) and $u\left(t_{0}\right)=u_{0}$ is given by

$$
u(t)=e_{p}\left(t, t_{0}\right) u_{0}+\int_{t_{0}}^{t} e_{p}(t, \sigma(\tau)) f(\tau) \Delta \tau
$$


(ii) Let $v_{0} \in \mathbb{R}$. The unique solution $v$ satisfying the right equation in (1.4) and $v\left(t_{0}\right)=v_{0}$ is given by

$$
v(t)=e_{\ominus p}\left(t, t_{0}\right) v_{0}+\int_{t_{0}}^{t} e_{\ominus p}(t, \tau) f(\tau) \Delta \tau .
$$

Motivated by the fact that (for differential equations) $u=1 / y$ solves a linear equation if $y$ is a solution of the logistic equation, we assume that $u$ is a solution of the first linear equation in (1.4), and then $y=1 / u$ satisfies

$$
y^{\Delta}=\left(\frac{1}{u}\right)^{\Delta}=-\frac{u^{\Delta}}{u u^{\sigma}}=-\frac{p u+f}{u u^{\sigma}}=-(p+f y) y^{\sigma}=[\ominus(p+f y)] y .
$$

Therefore we call the first equation in (1.5) (with $p+f y \in \mathcal{R}$ ) a logistic dynamic equation (or Verhulst equation). Similarly, we could start with a solution $v$ of the second linear equation in (1.4) and find that $x=1 / v$ satisfies

$$
x^{\Delta}=\left(\frac{1}{v}\right)^{\Delta}=-\frac{v^{\Delta}}{v v^{\sigma}}=\frac{p v^{\sigma}-f}{v v^{\sigma}}=\left(p-f x^{\sigma}\right) x=[p \ominus f x] x .
$$

We also call the second equation in (1.5) (with $f x \in \mathcal{R}$ ) a logistic dynamic equation. Throughout we assume $p \in \mathcal{R}$ and $f \in \mathrm{C}_{\mathrm{rd}}$. Note that if $u(t) \neq 0$ for all $t \in \mathbb{T}$, then $y=1 / u$ solves the first equation in (1.5) and automatically satisfies $p+f y \in \mathcal{R}$, as can be seen from the calculation

$$
1+\mu(p+f y)=\frac{u+\mu(p u+f)}{u}=\frac{u+\mu u^{\Delta}}{u}=\frac{u^{\sigma}}{u} \neq 0 .
$$

Similarly, if $v(t) \neq 0$ for all $t \in \mathbb{T}$, then $x=1 / v$ solves the second equation in (1.5) and satisfies

$$
1+\mu f x=\frac{v+\mu f}{v}=\frac{v^{\sigma}+\mu\left(f-v^{\Delta}\right)}{v}=\frac{v^{\sigma}+\mu p v^{\sigma}}{v}=\frac{v^{\sigma}(1+\mu p)}{v} \neq 0
$$

so that $f x \in \mathcal{R}$ follows.

Using Theorem 4.1, it is now easy to solve logistic equations as follows.

THEOREM 4.2. Suppose $p \in \mathcal{R}$ and $f \in \mathrm{C}_{\mathrm{rd}}$.

(i) Let $y_{0} \neq 0$. If

$$
u(t)=\frac{e_{p}\left(t, t_{0}\right)}{y_{0}}+\int_{t_{0}}^{t} e_{p}(t, \sigma(\tau)) f(\tau) \Delta \tau \neq 0 \quad \text { for all } \quad t \in \mathbb{T},
$$

then

$$
y(t)=\frac{1}{u(t)}=\frac{e_{\ominus p}\left(t, t_{0}\right)}{\frac{1}{y_{0}}+\int_{t_{0}}^{t} e_{\ominus p}\left(\sigma(\tau), t_{0}\right) f(\tau) \Delta \tau}
$$

solves the first equation in (1.5) and satisfies $y\left(t_{0}\right)=y_{0}$.

(ii) Let $x_{0} \neq 0$. If

$$
v(t)=\frac{e_{\ominus p}\left(t, t_{0}\right)}{x_{0}}+\int_{t_{0}}^{t} e_{\ominus p}(t, \tau) f(\tau) \Delta \tau \neq 0 \quad \text { for all } \quad t \in \mathbb{T},
$$


then

$$
x(t)=\frac{1}{v(t)}=\frac{e_{p}\left(t, t_{0}\right)}{\frac{1}{x_{0}}+\int_{t_{0}}^{t} e_{p}\left(\tau, t_{0}\right) f(\tau) \Delta \tau}
$$

solves the second equation in (1.5) and satisfies $x\left(t_{0}\right)=x_{0}$.

In applications (e.g., population dynamics) one often assumes that there exists a constant $N \neq 0$ such that

$$
p(t)=N f(t) \quad \text { for all } \quad t \in \mathbb{T} .
$$

For the remainder of this section we assume (4.1). Then we can evaluate the integral in the denominator of the solution given in Theorem 4.2 (ii) explicitly as

$$
\int_{t_{0}}^{t} e_{p}\left(\tau, t_{0}\right) f(\tau) \Delta \tau=\int_{t_{0}}^{t} e_{p}\left(\tau, t_{0}\right) \frac{p(\tau)}{N} \Delta \tau=\frac{1}{N}\left[e_{p}\left(t, t_{0}\right)-1\right] .
$$

Hence we obtain the following corollary from Theorem 4.2 (ii).

Corollary 4.3. Suppose $p \in \mathcal{R}$ and let $N \neq 0$ be a constant. Let $x_{0} \neq 0$. If

$$
\frac{1}{x_{0}}-\frac{1}{N}+\frac{e_{p}\left(t, t_{0}\right)}{N} \neq 0 \quad \text { for all } \quad t \in \mathbb{T},
$$

then

$$
x(t)=\frac{e_{p}\left(t, t_{0}\right)}{\frac{1}{x_{0}}-\frac{1}{N}+\frac{e_{p}\left(t, t_{0}\right)}{N}}=\frac{1}{\frac{1}{N}+\left(\frac{1}{x_{0}}-\frac{1}{N}\right) e_{\ominus p}\left(t, t_{0}\right)}
$$

satisfies $x\left(t_{0}\right)=x_{0}$ and solves

$$
x^{\Delta}=\frac{p x\left(1-\frac{x}{N}\right)}{1+\frac{\mu p}{N} x} \quad \text { with } \quad \frac{p x}{N} \in \mathcal{R} .
$$

REMARK 4.4. As is common in population dynamics, we call the function $p$ from Corollary 4.3 the intrinsic growth function, while we refer to $N$ as the saturation level or environmental carrying capacity. Note also that the functions $x_{1}(t) \equiv 0$ and $x_{2}(t) \equiv N$ are solutions of (4.3), the so-called equilibrium solutions (or critical solutions). If the starting value of $x$ is between these two solutions, i.e., $x_{0} \in(0, N)$, then $1 / x_{0}-1 / N>0$ and hence

$$
\frac{1}{x_{0}}-\frac{1}{N}+\frac{e_{p}\left(t, t_{0}\right)}{N}>\frac{e_{p}\left(t, t_{0}\right)}{N}
$$

so that

$$
0<x(t)<\frac{e_{p}\left(t, t_{0}\right)}{\frac{1}{N} e_{p}\left(t, t_{0}\right)}=N, \quad \text { provided } \quad p \in \mathcal{R}^{+} .
$$

Note that $p \in \mathcal{R}^{+}$ensures that $e_{p}\left(t, t_{0}\right)$ is positive (see [3, Theorem 2.48]). If $x_{0}>N$, then $1 / x_{0}-1 / N<0$ and hence

$$
x(t)>N, \text { provided } p \in \mathcal{R}^{+} .
$$


Assume now that $p(t)>0$ for all $t \in \mathbb{T}$. Then $p \in \mathcal{R}^{+}$. If $x_{0} \in(0, N)$, then $0<x(t)<N$ and

$$
x^{\Delta}=\frac{p x\left(1-\frac{x}{N}\right)}{1+\frac{\mu p}{N} x}>0
$$

so that $x$ is strictly increasing. Similarly, if $x_{0}>N$, then $x$ is strictly decreasing. In any case, if $x_{0} \neq 0$ and if $\mathbb{T}$ is unbounded above, then

$$
\lim _{t \rightarrow \infty} x(t)=N, \quad \text { provided } \quad \lim _{t \rightarrow \infty} e_{\ominus p}\left(t, t_{0}\right)=0 .
$$

EXAmPLE 4.5. Let $\mathbb{T}=\mathbb{R}$, let $\alpha$ be a constant, and consider the (continuous) logistic equation

$$
x^{\Delta}=[\alpha \ominus(\alpha x)] x, \quad \text { i.e., } \quad x^{\prime}=\alpha x(1-x) .
$$

Everything in Remark 4.4 applies to (4.4) (here $p(t) \equiv \alpha$ and $N=1$ ), and by Corollary 4.3 the solution of (4.4) with $x(s)=z$ (where $s \in \mathbb{R}$ and $z \in \mathbb{R} \backslash\{0\}$ ) is

$$
x(t)=\frac{e^{\alpha(t-s)}}{\frac{1}{z}-1+e^{\alpha(t-s)}}=\frac{1}{1+\left(\frac{1}{z}-1\right) e^{\alpha(s-t)}} .
$$

EXAmPLE 4.6. Let $\mathbb{T}=\mathbb{Z}$ and consider the (discrete) logistic equation

$$
x^{\Delta}=[t \ominus(t x)] x, \quad \text { i.e., } \quad \Delta x=\frac{t x(1-x)}{1+t x} .
$$

Everything in Remark 4.4 applies to (4.5) (here $p(t)=t$ and $N=1$ ), and by Corollary 4.3 the solution of (4.5) with $x(s)=z$ (where $s \in \mathbb{Z}$ and $z \in \mathbb{R} \backslash\{0\}$ ) is

$$
x(t)=\frac{\frac{t !}{s !}}{\frac{1}{z}+\frac{t !}{s !}-1}=\frac{1}{1+\left(\frac{1}{z}-1\right) \frac{s !}{t !}} .
$$

REMARK 4.7. One often finds an equation of the form

$$
y_{n+1}=\alpha y_{n}\left(1-\frac{y_{n}}{N}\right)
$$

referred to as a "logistic difference equation". Observe that this equation and our logistic difference equation introduced in this section are not the same. Our equation has the advantage that it is easily accessible with the methods used to study the "normal" logistic differential equation. Even though (4.6) appears as a natural analogue of the logistic differential equation, the results given in this section indicate that our equation "deserves" the name logistic equation rather than (4.6) (which is of course also worthy of study for its richness in dynamics). 
5. The Regressive Vector Space. For $\alpha \in \mathbb{R}$, we want to define the Bernoulli equation in such a way, that the substitution $\tilde{x}=x^{\alpha}$ transforms a solution $x$ of the Bernoulli equation into a solution $\tilde{x}$ of the logistic equation. To do so, we now find a formula for the derivative of $x^{\alpha}$. This task leads us in this section to the introduction of a circle dot multiplication $\odot$, which turns out to make $\left(\mathcal{R}^{+}, \oplus, \odot\right)$ into a vector space, the so-called regressive vector space. To begin with, we use the chain rule [3, Theorem 1.90] to calculate

$$
\tilde{x}^{\Delta}(t)=\left(x^{\alpha}\right)^{\Delta}(t)=x^{\Delta}(t) \int_{0}^{1} \alpha\left(x(t)+\mu(t) x^{\Delta}(t) h\right)^{\alpha-1} d h .
$$

If $x(t) \neq 0$, then

$$
\tilde{x}^{\Delta}(t)=\tilde{x}(t) \frac{x^{\Delta}(t)}{x(t)} \int_{0}^{1} \alpha\left(1+\mu(t) \frac{x^{\Delta}(t)}{x(t)} h\right)^{\alpha-1} d h .
$$

In order to have everything well defined, we want to assume that, if $\alpha \in \mathbb{R} \backslash \mathbb{N}$,

$$
1+\mu(t) \frac{x^{\Delta}(t)}{x(t)} h>0 \quad \text { for all } \quad t \in \mathbb{T} \quad \text { and all } \quad h \in[0,1] .
$$

It is easy to see that $x^{\Delta} / x \in \mathcal{R}^{+}$is sufficient for (5.2). Let us introduce the notation

$$
\mathcal{R}(\alpha):=\left\{\begin{array}{lll}
\mathcal{R} & \text { if } & \alpha \in \mathbb{N} \\
\mathcal{R}^{+} & \text {if } & \alpha \in \mathbb{R} \backslash \mathbb{N} .
\end{array}\right.
$$

Note that $p \in \mathcal{R}^{+}$implies that

$$
1+\mu(t) p(t) h>0 \quad \text { for all } \quad t \in \mathbb{T} \quad \text { and all } \quad h \in[0,1] .
$$

Definition 5.1. For $\alpha \in \mathbb{R}$ and $p \in \mathcal{R}(\alpha)$ we define $\alpha \odot p$ by (1.6).

REMARK 5.2. Note that $\alpha \odot p=\alpha p$ provided $\mathbb{T}=\mathbb{R}$.

THEOREM 5.3. Let $\alpha \in \mathbb{R}$. If $\alpha \in \mathbb{N}$, suppose that $x(t) \neq 0$ for all $t \in \mathbb{T}$. If $\alpha \notin \mathbb{N}$, suppose that $x(t) x^{\sigma}(t)>0$ for all $t \in \mathbb{T}$. Then the first formula in (1.8) holds.

Proof. First note that $1+\mu x^{\Delta} / x=x^{\sigma} / x$ implies $x^{\Delta} / x \in \mathcal{R}(\alpha)$. Then the statement follows from (5.1).

EXAMPLE 5.4. It is easy to see that

$$
2 \odot p=p \oplus p, \quad \frac{1}{2} \odot p=\frac{p}{1+\sqrt{1+\mu p}}, \quad \text { and } \quad 2 \odot\left(\frac{1}{2} \odot p\right)=p .
$$

REMARK 5.5. The three formulas

$$
\frac{(x y)^{\Delta}}{x y}=\frac{x^{\Delta}}{x} \oplus \frac{y^{\Delta}}{y}, \quad \frac{(x / y)^{\Delta}}{x / y}=\frac{x^{\Delta}}{x} \ominus \frac{y^{\Delta}}{y}, \quad \frac{\left(x^{\alpha}\right)^{\Delta}}{x^{\alpha}}=\alpha \odot \frac{x^{\Delta}}{x}
$$


could be used to define a logarithm as

$$
\log _{f}\left(t, t_{0}\right)=\int_{t_{0}}^{t} \frac{f^{\Delta}(\tau)}{f(\tau)} \Delta \tau
$$

but then the usual logarithm rules would not hold as we had formulas involving an additional integral, e.g.,

$$
\log _{x y}\left(t, t_{0}\right)=\log _{x}\left(t, t_{0}\right)+\log _{y}\left(t, t_{0}\right)+\int_{t_{0}}^{t} \frac{\mu(\tau) x^{\Delta}(\tau) y^{\Delta}(\tau)}{x(\tau) y(\tau)} \Delta \tau .
$$

It is still an open problem to define a logarithm that satisfies somehow "smoother" logarithm rules.

TheOREm 5.6. Let $\alpha \in \mathbb{R}$. If $p \in \mathcal{R}(\alpha)$, then $\alpha \odot p \in \mathcal{R}$. More precisely, the second formula in (1.8) holds.

Proof. Let $\alpha \in \mathbb{R}$ and $p \in \mathcal{R}(\alpha)$. Then

$$
1+\mu(\alpha \odot p)=1+\mu \alpha p \int_{0}^{1}(1+\mu p h)^{\alpha-1} d h=1+\int_{1}^{1+\mu p} \alpha s^{\alpha-1} d s=(1+\mu p)^{\alpha},
$$

where we used the substitution $s=1+\mu p h$. Therefore, $\alpha \odot p \in \mathcal{R}$. $\square$

The following result emphasizes again the importance of our circle dot multiplication introduced in Definition 5.1.

TheOrem 5.7. If $\alpha \in \mathbb{R}$ and $p \in \mathcal{R}(\alpha)$, then the third formula in (1.8) holds.

Proof. First note that $p \in \mathcal{R}(\alpha)$ implies $\alpha \odot p \in \mathcal{R}$ by Theorem 5.6. Now we let $t_{0} \in \mathbb{T}$ and put $y=e_{p}^{\alpha}\left(\cdot, t_{0}\right)$. Then $y\left(t_{0}\right)=1$ and by Theorem 5.3 (we skip the second argument $t_{0}$ )

$$
y^{\Delta}=\left(e_{p}^{\alpha}\right)^{\Delta}=\left(\alpha \odot \frac{e_{p}^{\Delta}}{e_{p}}\right) e_{p}^{\alpha}=(\alpha \odot p) y
$$

Hence $y$ solves the initial value problem

$$
y^{\Delta}=(\alpha \odot p)(t) y, \quad y\left(t_{0}\right)=1 .
$$

But this initial value problem has exactly one solution, namely $e_{\alpha \odot p}\left(\cdot, t_{0}\right)$.

We will use Theorem 5.7 to show that $\left(\mathcal{R}^{+}, \oplus, \odot\right)$ satisfies the axioms of a vector space. To do so, the following easy auxiliary result is helpful.

Lemma 5.8. Suppose $p, q \in \mathcal{R}$. If $e_{p}=e_{q}$, then $p=q$.

Proof. Differentiate both sides of $e_{p}=e_{q}$ to obtain this result.

Now we are ready to prove the main result of this section.

TheOrem 5.9. $\left(\mathcal{R}^{+}, \oplus, \odot\right)$ is a real vector space.

Proof. As is known (see e.g., [3, Lemma 2.47]), $\left(\mathcal{R}^{+}, \oplus\right)$ is an Abelian group. We now prove the remaining four vector space properties

$$
\alpha \odot(\beta \odot p)=(\alpha \beta) \odot p \quad \text { for all } \quad \alpha, \beta \in \mathbb{R}, p \in \mathcal{R}^{+} ;
$$




$$
1 \odot p=p \quad \text { for all } \quad p \in \mathcal{R}^{+}
$$

$$
\begin{aligned}
& \alpha \odot(p \oplus q)=(\alpha \odot p) \oplus(\alpha \odot q) \quad \text { for all } \quad p, q \in \mathcal{R}^{+}, \alpha \in \mathbb{R} ; \\
& (\alpha+\beta) \odot p=(\alpha \odot p) \oplus(\beta \odot p) \quad \text { for all } \quad \alpha, \beta \in \mathbb{R}, p \in \mathcal{R}^{+} .
\end{aligned}
$$

Clearly, (5.4) follows directly from Definition 5.1. For (5.3), (5.5), and (5.6) we use Theorem 5.7, Lemma 5.8, the rules (1.9) for the exponential function, and the "normal" rules of exponents as follows:

$$
e_{\alpha \odot(\beta \odot p)}=e_{\beta \odot p}^{\alpha}=\left(e_{p}^{\alpha}\right)^{\beta}=e_{p}^{\alpha \beta}=e_{(\alpha \beta) \odot p}
$$

implies (5.3),

$$
e_{\alpha \odot(p \oplus q)}=e_{p \oplus q}^{\alpha}=\left(e_{p} e_{q}\right)^{\alpha}=e_{p}^{\alpha} e_{q}^{\alpha}=e_{\alpha \odot p} e_{\alpha \odot q}=e_{(\alpha \odot p) \oplus(\alpha \odot q)}
$$

implies (5.5), and

$$
e_{(\alpha+\beta) \odot p}=e_{p}^{\alpha+\beta}=e_{p}^{\alpha} e_{p}^{\beta}=e_{\alpha \odot p} e_{\beta \odot p}=e_{(\alpha \odot p) \oplus(\beta \odot p)}
$$

implies (5.6).

Remark 5.10. Note that (5.4) of course holds for each $p \in \mathcal{R}$. Furthermore, if $\alpha, \beta \in \mathbb{N}$, then (5.3), (5.5), and (5.6) also hold for $p, q \in \mathcal{R}$.

REMARK 5.11. A referee pointed out that the circle dot product allows the representation $(\alpha \odot p)(t)=\lim _{h \rightarrow \mu(t)} \frac{(1+h p(t))^{\alpha}-1}{h}$, but we are not using this formula here.

6. Bernoulli Equations. Now we let $\alpha \in \mathbb{R} \backslash\{0\}$ and consider the Bernoulli equation (1.7). Note that (1.7) is in the form of the second equation in (1.5) if $\alpha=1$. Introducing $\tilde{x}=x^{\alpha}$, we find if $x$ never vanishes and solves (1.7), then

$$
\frac{\tilde{x}^{\Delta}}{\tilde{x}}=\frac{\left(x^{\alpha}\right)^{\Delta}}{x^{\alpha}}=\alpha \odot \frac{x^{\Delta}}{x}=\alpha \odot\left[p \ominus\left(\frac{1}{\alpha} \odot f x^{\alpha}\right)\right]=(\alpha \odot p) \ominus f \tilde{x},
$$

where we used Theorem 5.3 and the vector space properties (5.3) and (5.5). Hence $\tilde{x}$ solves the logistic equation

$$
\tilde{x}^{\Delta}=[(\alpha \odot p) \ominus(f \tilde{x})] \tilde{x} .
$$

Equation (6.1) is of the form (1.5), so that we may apply Theorem 4.2 (ii) (use also Theorem 5.7) to find $\tilde{x}$ as

$$
\tilde{x}(t)=\frac{e_{p}^{\alpha}\left(t, t_{0}\right)}{\frac{1}{\tilde{x}_{0}}+\int_{t_{0}}^{t} e_{p}^{\alpha}\left(\tau, t_{0}\right) f(\tau) \Delta \tau} .
$$

It is hence easy to show the following result.

Theorem 6.1. Suppose $\alpha \in \mathbb{R} \backslash\{0\}, p \in \mathcal{R}(\alpha)$, and $f \in \mathrm{C}_{\mathrm{rd}}$. Let $x_{0} \neq 0$. If

$$
\frac{1}{x_{0}^{\alpha}}+\int_{t_{0}}^{t} e_{p}^{\alpha}\left(\tau, t_{0}\right) f(\tau) \Delta \tau>0 \quad \text { for all } \quad t \in \mathbb{T},
$$


then

$$
x(t)=\frac{e_{p}\left(t, t_{0}\right)}{\left[\frac{1}{x_{0}^{\alpha}}+\int_{t_{0}}^{t} e_{p}^{\alpha}\left(\tau, t_{0}\right) f(\tau) \Delta \tau\right]^{1 / \alpha}}
$$

solves the Bernoulli equation (1.7).

EXAmPle 6.2. Consider the initial value problem

$$
x^{\prime}=-x+t x^{4}, \quad x(0)=1 .
$$

Note that the differential equation in $(6.2)$ can be rewritten as a dynamic (with $\mathbb{T}=\mathbb{R}$ ) Bernoulli equation

$$
x^{\Delta}=\left[p \ominus\left(\frac{1}{\alpha} \odot f x^{\alpha}\right)\right] x \quad \text { with } \quad p(t) \equiv-1, f(t)=-3 t, \alpha=3 .
$$

According to Theorem 6.1 (with $t_{0}=0$ and $x_{0}=1$ ), the solution of $(6.2)$ is

$$
x(t)=\frac{e_{p}\left(t, t_{0}\right)}{\left[\frac{1}{x_{0}^{\alpha}}+\int_{t_{0}}^{t} e_{p}^{\alpha}\left(\tau, t_{0}\right) f(\tau) \Delta \tau\right]^{1 / \alpha}}=\frac{e^{-t}}{\left[\frac{2}{3}+\left(t+\frac{1}{3}\right) e^{-3 t}\right]^{1 / 3}} .
$$

(We point out that $x$ is not a solution of (6.2) on the whole time scale.)

ExAmple 6.3. Consider the initial value problem

$$
\Delta x=\frac{5 x-x^{3}+5 x \sqrt{1+x^{2}}}{1+x^{2}+\sqrt{1+x^{2}}}, \quad x(0)=1 .
$$

Note that

$$
\frac{5-x^{2}+5 \sqrt{1+x^{2}}}{1+x^{2}+\sqrt{1+x^{2}}}=5 \ominus \frac{x^{2}}{1+\sqrt{1+x^{2}}}=5 \ominus\left(\frac{1}{2} \odot x^{2}\right)
$$

implies that the difference equation in (6.3) can be rewritten as a dynamic (with $\mathbb{T}=\mathbb{Z})$ Bernoulli equation

$$
x^{\Delta}=\left[p \ominus\left(\frac{1}{\alpha} \odot f x^{\alpha}\right)\right] x \quad \text { with } \quad p(t) \equiv 5, f(t) \equiv 1, \alpha=2 .
$$

According to Theorem 6.1 (with $t_{0}=0$ and $x_{0}=1$ ), the solution of $(6.3)$ is

$$
x(t)=\frac{e_{p}\left(t, t_{0}\right)}{\left[\frac{1}{x_{0}^{\alpha}}+\int_{t_{0}}^{t} e_{p}^{\alpha}\left(\tau, t_{0}\right) f(\tau) \Delta \tau\right]^{1 / \alpha}}=\frac{6^{t} \sqrt{35}}{\sqrt{34+36^{t}}} .
$$


7. Riccati Equations. In this section we consider the Riccati equation

$$
z^{\Delta}+q(t)+r(t) z^{\sigma}+\frac{z^{2}}{p(t)+\mu(t) z}=0,
$$

where $p, q \in \mathrm{C}_{\mathrm{rd}}, r \in \mathcal{R}$, and $p(t) \neq 0$ for all $t \in \mathbb{T}$. Note that (7.1) can be written in the form (1.10). We now assume that $z_{1}$ is a known solution of (1.10). We pick any other solution $z$ of (1.10) and consider the difference $y=z-z_{1}$. It follows that

$$
\begin{aligned}
y^{\Delta} & =-q-r z^{\sigma}-p\left(\frac{z}{p}\right)^{(2)}+q+r z_{1}^{\sigma}+p\left(\frac{z_{1}}{p}\right)^{(2)} \\
& =-r y^{\sigma}+p\left[\left(\frac{z_{1}}{p}\right)^{(2)}-\left(\frac{z}{p}\right)^{(2)}\right] .
\end{aligned}
$$

To continue this calculation, the following lemma is useful and can be proved by a direct computation.

Lemma 7.1. For $p, q \in \mathcal{R}$ we have $p^{(2)}-q^{(2)}=[\ominus(p \oplus q)](q-p)$.

We may use Lemma 7.1 to continue our calculation from above as follows:

$$
\begin{aligned}
y^{\Delta} & =-r y^{\sigma}+p\left[\left(\frac{z_{1}}{p}\right)^{2}-\left(\frac{z}{p}\right)^{2}\right] \\
& =-r y^{\sigma}+p\left[\ominus\left(\frac{z_{1}}{p} \oplus \frac{z}{p}\right)\right]\left(\frac{z}{p}-\frac{z_{1}}{p}\right) \\
& =-r y^{\sigma}+y\left[\ominus\left(\frac{z_{1}}{p} \oplus \frac{z}{p}\right)\right] .
\end{aligned}
$$

Denoting $s=\ominus\left(\frac{z_{1}}{p} \oplus \frac{z}{p}\right)$, we continue to find

$$
y^{\Delta}=-r y^{\sigma}+y s=-r y-r \mu y^{\Delta}+y s=y(s-r)-r \mu y^{\Delta}
$$

and therefore (solve this last equation for $y^{\Delta}$ )

$$
y^{\Delta}=y \frac{s-r}{1+\mu r}=y(s \ominus r) .
$$

Now, apply the formula

$$
a \oplus(b+c)=a+b+c+\mu a b+\mu a c=(a \oplus b)+c(1+\mu a)
$$

to find

$$
\begin{aligned}
\ominus(s \ominus r) & =\frac{z_{1}}{p} \oplus \frac{z}{p} \oplus r=\frac{z_{1}}{p} \oplus r \oplus\left(\frac{z_{1}}{p}+\frac{y}{p}\right) \\
& =\left[\left(2 \odot \frac{z_{1}}{p}\right) \oplus r\right]+\frac{y}{p}\left[1+\mu\left(\frac{z_{1}}{p} \oplus r\right)\right]=g+f y
\end{aligned}
$$

where

$$
f=\frac{1+\mu\left(r \oplus \frac{z_{1}}{p}\right)}{p} \quad \text { and } \quad g=r \oplus\left(2 \odot \frac{z_{1}}{p}\right)
$$


Hence, using (7.2), we find that $y$ satisfies the equation

$$
y^{\Delta}=[\ominus(g(t)+f(t) y)] y .
$$

Equation (7.4) is a logistic equation as in (1.5). Hence the following result holds.

ThEOREM 7.2. Suppose $z_{1}$ is a solution of the Riccati equation (1.10). Define $f$ and $g$ by (7.3) and let $y$ be a solution of the logistic equation (7.4). Then $z=z_{1}+y$ is also a solution of the Riccati equation (1.10).

Corollary 7.3. Suppose $z_{1}$ is a solution of the Riccati equation (1.10). Define $f$ and $g$ by (7.3). Then a solution $z$ of (1.10) satisfying $z\left(t_{0}\right)=z_{0}$ is given by

$$
z(t)=z_{1}(t)+\frac{e_{g}\left(t_{0}, t\right)}{\frac{1}{z_{0}-z_{1}\left(t_{0}\right)}+\int_{t_{0}}^{t} e_{g}\left(t_{0}, \sigma(\tau)\right) f(\tau) \Delta \tau},
$$

provided none of the denominators is zero.

Proof. This follows from Theorem 4.2, where solutions of (1.5) are given.

EXAMPLE 7.4. We consider the equation

$$
z^{\Delta}+[\ominus(-t)] z^{\sigma}+\frac{z^{2}}{\mu(t) z-\frac{1}{t}}=0 .
$$

Equation (7.5) is a Riccati equation of the form (7.1) with coefficients $p(t)=-\frac{1}{t}$, $q(t)=0$, and $r(t)=\ominus(-t)$. By examination, $z_{1}(t) \equiv 1$ solves (7.5). We have

$$
\frac{z_{1}(t)}{p(t)}=-t \quad \text { and } \quad\left(r \oplus \frac{z_{1}}{p}\right)(t)=[\ominus(-t)] \oplus(-t)=0
$$

so that $f$ and $g$ from (7.3) are given by $f(t)=g(t)=-t$. Hence, by Corollary 7.3, we find that another solution of $(7.5)$ is given by

$$
z(t)=\frac{1}{1-\left(1-\frac{1}{z_{0}}\right) e_{\ominus(-t)}\left(t, t_{0}\right)},
$$

provided none of the demoninators is zero. This solution $z$ satisfies $z\left(t_{0}\right)=z_{0}$.

8. Clairaut Equations. The Clairaut dynamic equation appears as in (1.11), where $f: \mathbb{R} \rightarrow \mathbb{R}$ is some continuously differentiable function. We proceed to treat (1.11) as its continuous analogue $y=t y^{\prime}+f\left(y^{\prime}\right)$ and perform the substitution $v=y^{\Delta}$. Then

$$
y(t)=t v(t)+f(v(t)) .
$$

Differentiating (8.1) and using [3, Theorem 1.90], we conclude

$$
v(t)=y^{\Delta}(t)=\sigma(t) v^{\Delta}(t)+v(t)+v^{\Delta}(t) \int_{0}^{1} f^{\prime}\left(v(t)+h \mu(t) v^{\Delta}(t)\right) d h
$$

so that

$$
0=v^{\Delta}(t)\left\{\sigma(t)+\int_{0}^{1} f^{\prime}\left(v(t)+h \mu(t) v^{\Delta}(t)\right) d h\right\}
$$


(Note that differentiating both sides of (8.1) can introduce extraneous solutions.) Hence we obtain the following result.

THEOREM 8.1. For any constant $c \in \mathbb{R}$,

$$
y(t)=c t+f(c)
$$

is a solution of (1.11). Further solutions of (1.11) may be obtained by solving

$$
\int_{0}^{1} f^{\prime}\left(v(t)+h \mu(t) v^{\Delta}(t)\right) d h=-\sigma(t), \quad y^{\Delta}=v .
$$

EXAMPLE 8.2. Consider the equation

$$
y=t y^{\Delta}+\left(y^{\Delta}\right)^{2}
$$

which is of the form (1.11) with $f(x)=x^{2}$. By (8.2) of Theorem 8.1, we find that $y(t)=c t+c^{2}$ is a solution of $(8.4)$, where $c \in \mathbb{R}$ is an arbitrary constant. With $v=y^{\Delta}$, the left hand side of (8.3) becomes

$$
\int_{0}^{1} f^{\prime}\left(v(t)+h \mu(t) v^{\Delta}(t)\right) d h=2 \int_{0}^{1}\left(v(t)+h \mu(t) v^{\Delta}(t)\right) d h=2 v(t)+\mu(t) v^{\Delta}(t) .
$$

Hence we must solve

$$
2 v+\mu(t) v^{\Delta}=-\sigma(t), \quad \text { i.e., } \quad v+v^{\sigma}=-\sigma(t)
$$

If $\mathbb{T}=\mathbb{R}$, then $(8.5)$ becomes $2 v(t)=-t$, and therefore $y(t)=-\frac{t^{2}}{4}$ is also a solution of (8.4). Now we assume that

$$
\mathbb{T}=\left\{t_{k}: k \in \mathbb{N}_{0}\right\} \quad \text { with } \quad t_{0}<t_{1}<t_{2}<\ldots
$$

Then we may divide $(8.5)$ by $\mu(t)$ to arrive at

$$
v^{\Delta}=\frac{2}{\mu(t)} v^{\sigma}+\frac{\sigma(t)}{\mu(t)}
$$

which is an equation as in (1.4) with $p=-2 / \mu$ and $f=\sigma / \mu$. By Theorem 4.1, a solution is given by (note that $\ominus(-2 / \mu)=-2 / \mu$ )

$$
v(t)=e_{-2 / \mu}\left(t, t_{0}\right) v_{0}+\int_{t_{0}}^{t} e_{-2 / \mu}(t, \tau) \frac{\sigma(\tau)}{\mu(\tau)} \Delta \tau .
$$

We find

$$
v\left(t_{m}\right)=(-1)^{m} v_{0}+\sum_{k=0}^{m-1}(-1)^{m-k} t_{k+1}=(-1)^{m} c-(-1)^{m}\left[\alpha+\sum_{k=1}^{m}(-1)^{k} t_{k}\right],
$$

where we replaced $v_{0}$ by $c-\alpha$. The value of the above sum depends on the time scale, and we will do the calculation for a few time scales that satisfy (8.6). First,

$$
\mathbb{T}=\mathbb{N}_{0} \quad \text { implies } \quad \sum_{k=1}^{m}(-1)^{k} t_{k}=(-1)^{m}\left(\frac{m}{2}-\frac{1}{4}\right)-\frac{1}{4} .
$$


Now we check for which values of $\alpha$ we do have indeed a solution (as extraneous solutions could have been introduced) and find $\alpha=1 / 4$ and conclude that

$$
y(t)=\frac{(-1)^{t}}{2} c-\frac{t(t-1)}{4}-\frac{t}{4}+\left(\frac{1}{16}+c^{2}\right)=\left(c(-1)^{t}-\frac{1}{4}\right)^{2}-\frac{t^{2}}{4}
$$

is also a solution of (8.4) for any $c \in \mathbb{R}$ (see also [1, Example 3.1.1]). Next,

$$
\mathbb{T}=q^{\mathbb{N}_{0}} \quad \text { implies } \quad \sum_{k=1}^{m}(-1)^{k} t_{k}=(-1)^{m} \frac{q^{m+1}}{q+1}-\frac{q}{q+1},
$$

so we choose $\alpha=q /(q+1)$ and derive that

$$
y(t)=\frac{1-q}{1+q} c t(-1)^{\log _{q} t}-\frac{q t^{2}}{(q+1)^{2}}+c^{2}=\left(c(-1)^{\log _{q} t}-\frac{(q-1) t}{2(q+1)}\right)^{2}-\frac{t^{2}}{4}
$$

is also a solution of (8.4) for any $c \in \mathbb{R}$. Finally, we note that

$$
\mathbb{T}=\mathbb{N}_{0}^{2} \quad \text { implies } \quad \sum_{k=1}^{m}(-1)^{k} t_{k}=(-1)^{m} \frac{m(m+1)}{2},
$$

and hence we choose $\alpha=0$ and find that

$$
y(t)=-c \sqrt{t}(-1)^{\sqrt{t}}-\frac{t^{2}}{4}+\frac{t}{4}+c^{2}=\left(c(-1)^{\sqrt{t}}-\frac{\sqrt{t}}{2}\right)^{2}-\frac{t^{2}}{4}
$$

is also a solution of (8.4) for any $c \in \mathbb{R}$.

Acknowledgement. We would like to thank the three referees who pointed out some directions on how to improve this paper (see e.g., Remarks 2.9 and 5.11).

\section{REFERENCES}

[1] R. P. Agarwal, Difference Equations and Inequalities. Marcel Dekker, Inc., New York, 1992.

[2] F. M. Atici And M. Atici, Analysis of divide-and-conquer algorithms on time scales, 2003. In preparation.

[3] M. Bohner And A. Peterson, Dynamic Equations on Time Scales: An Introduction with Applications, Birkhäuser, Boston, 2001.

[4] S. Hilger, Analysis on measure chains - a unified approach to continuous and discrete calculus, Results Math., 18:18-56, 1990.

[5] B. Kaymakçalan, V. Lakshmikantham, and S. Sivasundaram, Dynamic Systems on Measure Chains, volume 370 of Mathematics and its Applications, Kluwer Academic Publishers Group, Dordrecht, 1996. 